\title{
Preface
}

The 2020 5th International Conference on Advances in Energy and Environment Research (ICAEER 2020) was held in the form of online conference on September 18-20, 2020, which should be held in Shanghai, China as planned. Due to the impact of COVID-19 and travel restrictions, ICAEER 2020 was held via online collaboration tool Zoom, which is different from the traditional way. This can not only effectively reduce people gathering and prevent COVID-19, but also enable the scholars to make academic communications as scheduled. ICAEER 2020 aimed to provide a flexible platform for experts and scholars in the field of Energy and Environment to disseminate the latest research achievements and advanced technologies, as well as to promote the cooperation between each other.

Firstly, we were honored to invite Prof. Hexing Li, President of Shanghai University of Electric Power, China to make an opening speech for the conference. Prof.Li has been president of Shanghai University of Electric Power since January 2011. He has won the honorary titles of "The National Science Fund for Distinguished Young Scholars ", "Shanghai Leading Talents Program" and so on. And we were also honored to invite Prof. Jiang Wu, Shanghai University of Electric Power, China as conference co-chair to host the conference. During the conference, the conference model was divided into three sessions, including keynote speeches, oral presentations and online Q\&A discussion.

In the keynote speeches session, keynote speakers were each allocated 30-40 minutes to hold their speeches. We were honored to invite seven distinguished experts as keynote speakers to present their insightful speeches. Dr.Rohitha Weerasinghe from University of the West of England, England, made a speech about Procedural Representation Based Product Data Exchange. Prof. Minghou Xu from Huazhong University of Science and Technology, China, shared his study on Fine particulate matter from solid fuel combustion: fundamental research and practice. Prof. Qiang Wang from Beijing Forestry University, China and his speech title is: Inter-mediate and high temperature solid $\mathrm{CO} 2$ capture materials. Prof. Qiang Lu from North China Electric Power University, China, held a speech on the topic of Production of fuels and chemicals from pyrolysis of biomass and organic solid wastes: mechanism, technologies and applications. Prof. Zhongzhu Qiu from Shanghai University of Electric Power, China, his speech is about Micro-encapsulated phase change material (MPCM) slurries: Characterization and applications. Prof. Zhuoying Tan from University of Science and Technology Beijing, China, made a speech about Ecosuppression and intelligent control techniques on open heavy dust based on big data. And Prof.Jiang Wu from Shanghai University of Electric Power, China, his speech title is Mechanism and application of photocatalytic oxidation technology in the control of heavy metals in Flue Gas.

In the oral presentation's session, some scholars were given about 10-15 minutes to perform their oral presentations one by one. we were honored to invite Prof. Chih-Huang Weng, I-Shou University and Prof. Qian Xu, Jiangsu University as Co-Chairs of oral presentation's session. There were thirty scholars to present their speech on the session. In the Q\&A session, all participants were invited to join in academic groups to discuss and explore the academic issues after the presentations. The discussion session will last 30 minutes.

The proceedings collected the latest research and results in the field of Energy and Environment. The topic of this collection is related to Energy Engineering, Power Engineering, Pollution Control, Environmental Engineering and so on. All manuscripts published in the proceeding have been through rigorous review and process to meet the requirements of International publication standard. 
Finally, we would like to express our sincere gratitude to the Chairman, the distinguished keynote speakers, as well as all the participants. Because of your enthusiastic participation, the conference was successfully held as planned. Thanks to the information technology, the online conference made it easier for more people join in our conference. We had about 250 attendees this time, who are from China, Ukraine, Thailand, UK, Russia, Singapore and more countries and districts. We are expecting more and more experts and scholars from all over the world to join this international event next year. We also want to thank the publisher for publishing the proceedings. We are grateful to all those who have contributed to the success of ICAEER 2020. We hope that all participants and other interested readers benefit scientifically from the proceedings.

The Organizing Committee of ICAEER 2020 


\section{Conference Committees}

\section{Conference Chair}

Prof. Jiang Wu, Shanghai University of Electric Power

Prof. Chih-Huang Weng, I-Shou University

Dr. Rohitha Weerasinghe, University of the West of England

\section{Local Committee}

Prof. Qunzhi Zhu, Shanghai University of Electric Power

Prof. Weiguo Pan, Shanghai University of Electric Power

Prof. Minghou Xu, Huazhong University of Science and Technology

Prof. Xinyuan Zhu, Shanghai Jiaotong University

Prof. Qiang Wang, Beijing Forestry University

Prof. Yaji Huang, Southeast University

Prof. Ping Lu, Nanjing Normal University

Prof. Lin Dong, Nanjing University

Prof. Weidong Fan, Shanghai Jiaotong University

Prof. Dong Liu, Nanjing University of Science and Technology

Prof. Chunbo Wang, North China Electric Power University

Prof. Qiang Lu, North China Electric Power University

Prof. Boxiong Shen, Hebei University of Technology

Prof. Zhongzhu Qiu, Shanghai University of Electric Power

Prof. Hongbo Ren, Shanghai University of Electric Power

Prof. Tao Song, Nanjing Normal University

Prof. Binlin Dou, University of Shanghai for Science and Technology

Prof. Yufeng Duan, Southeast University

\section{International Technical Committee}

Prof. Chun Yang, Nanyang Technological University, Singapore

Academician of Japan Engineering Academy/Prof. Qingqing Ni, National Shinshu University, Japan

A/Prof./Dr. Jian Zong, Shanghai Institute of Technology, China

Prof. Yulong Ding, University of Birmingham Centre for Energy Storage, UK

Prof/Dr. Yonggao Yin, Southeast University, China

Dr. Sebastien Martinet, CEA-LITEN, France

A/Prof. Yucheng Lan, Morgan State University, USA

Prof. Jianxun Jin, Tianjin University, China

Dr. Priyanshu Verma, Bansal Institute of Engineering and Technology, India

Prof. Zhaode Zhang, Zhejiang Ocean University, China

Dr. PHAM THI LOAN, Haiphong University, Vietnam

Prof. Yung-Jaan Lee, Chung-Hua Institution for Economic Research

Prof. Haşim Altan, Final International University, Cyprus

Prof. Ben Cheikh Hamida, Ammar Telidji University, Algeria

Prof. Jiake Li, Xi' an University of Technology, China

A/Prof. Roberto Gomez, Universidad Nacional Autonoma de Mexico, Mexico

Prof. Fangming Chen, Hubei University, China

A/Prof. Mário A. A. Cunha,Federal University of Technology - Paraná (UTFPR),Brazil

Dr.Abdeen Omer,Energy Research Institute (ERI),United Kingdom 